\title{
Dietary linseed supplementation affects the fatty acid composition of the $s n-2$ position of triglycerides in sheep milk
}

\author{
A. Serra, ${ }^{*}{ }^{1}{ }^{1}$ G. Conte, ${ }^{*} \dagger$ F. Ciucci, ${ }^{*}$ E. Bulleri, ${ }^{*}$ L. Corrales-Retana, ${ }^{*}$ A. Cappucci, ${ }^{*}$ A. Buccioni, $\neq$ and M. Mele ${ }^{*} \dagger$ \\ *Department of Agriculture, Food and Environment, and \\ †Research Center of Nutraceuticals and Food for Health, University of Pisa, via del Borghetto 80, 56124 Pisa, Italy \\ $\ddagger$ Agrifood Production and Environmental Sciences, section of Animal Science, University of Florence, via delle Cascine 23, 50144 Florence, Italy
}

\begin{abstract}
In the human intestine, lipids are absorbed as $s n-2$ monoglycerides (sn-2, also named $\beta$-position), produced mainly by pancreatic lipases, which hydrolysate the triglyceride molecule in positions 1 and 3 (sn-1,3, $\alpha$-position). The fatty acids esterified in $s n-2$ are thus preferentially absorbed, which means that the bioavailability of a single fatty acid is affected by its position on the triglyceride. This experiment is carried out with the milk used to make cheese applied in a study with $42 \mathrm{hu}-$ man volunteers. In that study the authors detected an improvement in the blood lipid profile. The aim of the present study was to examine the effectiveness of this kind of cheese in improving human health by studying how linseed supplementation affects the milk fatty acid composition of the 3 different triglyceride positions and thus the fatty acid bioavailability. The $s n-2$ were obtained by reacting total milk lipids with swine pancreatic lipase. The milk came from 24 sheep fed a control diet and 24 sheep fed a diet containing $200 \mathrm{~g}$ of extruded linseed per day. The sn-2 were separated by thin-layer chromatography. The fatty acid composition of total lipids and $s n$-2 was obtained by a gas chromatographyflame ionization detector apparatus equipped with a high polar $100 \mathrm{~m}$ length capillary column. The bioavailability of the fatty acids was evaluated by a putative preferential intestinal absorption index (PPIAi), where PPIAi $<0$ indicated a disadvantageous nutritional condition and PPIAi $>0$ indicated a preferential intestinal absorption. With regard to the fatty acid composition of triglycerides, the linseed group showed a significantly higher content of both linolenic acid and rumenic acid compared with the control. As a consequence of linseed supplementation, the linolenic and rumenic acid content esterified in the $\beta$-position increased greatly. This was highlighted by the PPIAi. The results of the
\end{abstract}

Received November 23, 2017.

Accepted April 6, 2018.

${ }^{1}$ Corresponding author: andrea.serra@unipi.it present study suggest that the linolenic acid and conjugated linoleic acid affinity for lyso-phosphatidic acid acyl-transferase increased with its tissue availability. Key words: $s n-2$ monoglyceride fatty acid composition, linseed supplementation, sheep milk

\section{INTRODUCTION}

Several studies have shown that animal-derived food provides various healthy substances (named nutraceuticals) for the human body (Chen et al., 2006; Santini et al., 2017). Many of these substances belong to lipids, some of which are fatty acids (Blondeau, 2016). The content of bioactive substances in food can be increased using a rearing system (Serra et al., 2009), animal diet (Mele et al., 2011), or nutraceutical supplementation (Ghazal et al., 2014). Using one or more of these 3 strategies, it is possible to obtain fortified, pharm, designer, or more generally, functional foods. The effectiveness of this kind of food depends on the amount of bioactive fatty acids provided and their bioavailability. The literature data highlight that the effectiveness of a single bioactive fatty acid also depends on the overall fatty acid composition of the food. For instance, in a previous study, we obtained a significant natural enrichment in CLA (2.50 g/100 g of fat) and $\alpha$-linolenic acid (ALA, $2.10 \mathrm{~g} / 100$ of fat) of milk and cheese from sheep fed with a diet supplemented with linseed (Mele et al., 2011).

Ninety grams per day of the cheese from the milk used in this study was tested in a human trial including 42 dyslipidemic volunteers (19 males and 23 females). The cheese from the animals fed linseed increased the plasma concentration of PUFA n-3 fatty acids (including eicosapentaenoic fatty acid, C20:5n-3; EPA), CLA, and vaccenic acid (VA) and reduced the low-density lipoprotein (LDL) cholesterol, and various endocannabinoids such as 2-arachidonoyl glycerol and anandamide (Pintus et al., 2013). Thus, this kind of cheese improved the human lipid blood profile via an ALA intake of about $600 \mathrm{mg} / \mathrm{d}$. 
Table 1. Chemical composition of feed, diet, and milk (g/100 g of raw matter), SCC, and milk daily production $(\mathrm{MDI})^{1}$

\begin{tabular}{|c|c|c|c|c|c|c|c|}
\hline \multirow[b]{3}{*}{ Item } & \multicolumn{3}{|c|}{ Feed } & & & & \\
\hline & \multirow[b]{2}{*}{ Hay } & \multicolumn{2}{|c|}{ Concentrate } & \multicolumn{2}{|c|}{ Diet } & \multicolumn{2}{|c|}{ Milk } \\
\hline & & $\mathrm{CON}$ & LIN & $\mathrm{CON}$ & LIN & $\mathrm{CON}$ & LIN \\
\hline DM & 87.60 & 85.31 & 84.17 & 90.0 & 90.0 & 16.6 & 16.8 \\
\hline Total lipids & 2.4 & 2.9 & 9.6 & 2.6 & 8.7 & 5.6 & 5.5 \\
\hline $\mathrm{CP}$ & 8.7 & 17.7 & 17.9 & 16.1 & 15.9 & 5.3 & 5.4 \\
\hline Ash & 6.7 & 7.8 & 8.9 & 7.0 & 7.6 & 0.9 & 1.0 \\
\hline $\mathrm{NDF}$ & 49.3 & 26.4 & 21.0 & 37.0 & 36.9 & - & - \\
\hline $\mathrm{NSC}$ & 20.5 & 32.7 & 25.6 & 32.3 & 30.2 & - & - \\
\hline $\mathrm{NE}_{\mathrm{L}}(\mathrm{kcal})$ & 111.3 & 126.0 & 143.0 & 134.6 & 139.2 & - & - \\
\hline Lactose & - & - & - & - & - & 4.8 & 4.9 \\
\hline $\mathrm{SCC} \times 1.000$ & - & - & - & - & - & 286 & 283 \\
\hline MDI (g) & & & & & & 640.8 & 648.8 \\
\hline
\end{tabular}

${ }^{1}$ Treatments: $\mathrm{CON}=$ control; $\mathrm{LIN}=$ linseed

In contrast, Sofi et al. (2010) obtained a reduction in blood inflammatory parameters (including IL-6, IL-8, and $\mathrm{TNF}-\alpha$ ) in dyslipidemic subjects via a cheese that provided an even lower CLA and ALA content (150 and $60 \mathrm{mg} / \mathrm{d}$, respectively). However, this cheese did not lower the LDL cholesterol. Dawczynski et al. (2010 and 2013) detected a reduction in cardiovascular risk and pro-inflammatory eicosanoids in humans receiving yogurt supplemented with fish oil and rapeseed oil (3 $\mathrm{g} / \mathrm{d}$ of n-3 PUFA).

This evidence suggests that the effectiveness of a bioactive substance depends, not only on the amount in the food itself, but also on its bioavailability.

The majority of fatty acids in food are contained in triacylglycerols (TG), which are preferentially absorbed in the duodenum as $s n-2$ monoglycerides $(\boldsymbol{s n}-\mathbf{2}$, $\beta$-position), after the hydrolysis of fatty acids esterified in $\alpha$-position $(\boldsymbol{s n - 1 , 3 )}$ by the action of gastric and, above all, enteric lipases ( $\mathrm{Mu}$ and $\mathrm{H} \varnothing \mathrm{y}, 2004)$. Thus, with respect to the $\alpha$-position, the $\beta$-position represents a preferential nutritional condition that may affect fatty acid bioavailability (Ramírez et al., 2001) and, in turn, the effectiveness and economic sustainability of the animal lipid supplementation strategy.

In the present experiment, milk fat samples were obtained from the same milk used to make the control and enriched cheese for the clinical trial carried out by Pintus et al. (2013). To examine the effectiveness of this cheese in improving human health, we studied how linseed supplementation affects the fatty acid composition of the 3 triglyceride positions of milk. The aim was to provide some initial findings regarding the fatty acid bioavailability of enriched milk and cheese and to highlight the effectiveness of the adopted approach.

\section{MATERIALS AND METHODS}

\section{Animals and Milk Samples}

The experiment was carried out on 48 Sarda ewes divided into 2 groups: the control $(\mathbf{C O N}$, live weight $38.17 \mathrm{~kg} \pm 4.16$ ) and linseed (LIN, live weight $38.05 \mathrm{~kg}$ \pm 4.76 ), which were fed with hay (a mixture of Avena sativa L., Lolium multiflorum L., and Trifolium repens L.) and, respectively, with $800 \mathrm{~g} / \mathrm{d}$ of a concentrate containing $32 \%$ dry starch matter and $700 \mathrm{~g} / \mathrm{d}$ of a concentrate (Omega-Lin, Mignini, Assisi, Italy) containing $30 \%$ dry extruded linseed, thus providing $45 \mathrm{~g}$ of total linolenic acid. The feed was offered to the animals twice a day. The chemical composition of the feed and diets are listed in Table 1.

After about 9 wk of milking (CON, $107.17 \pm 6.83$ d; LIN, $106.42 \pm 7.06 \mathrm{~d})$, a milk sample was taken from each animal (obtained by mixing a sample of each of 2 daily milkings), which was immediately refrigerated and then stored at $-20^{\circ} \mathrm{C}$ until the analysis. Milk chemical composition, SCC, and milk daily production are shown in Table 1.

\section{Analysis}

Total lipids (TL) were extracted according to Serra et al. (2009). The $s n-2$ were obtained as follows: TL were placed on a $20 \times 20 \mathrm{~cm}$ silica gel-60 TLC plate (Merck, Darmstadt, Germany) and eluted with hexanediethyl ether to separate the TG. A chromatographic run was performed at $4^{\circ} \mathrm{C}$ in a refrigerator. After drying, the TLC plate was sprayed with a solution of 2,7 dichlorofluorescein (Merck) in ethanol $(0.2 \% \mathrm{wt} / \mathrm{vol})$. 
The spot corresponding to the TG was scraped, and the TG were extracted with diethyl ether.

Some of the TG were trans-esterified according to Christie (1982), the rest was used to obtain $s n-2$ as follows: Tris ( $1 \mathrm{M}$ at $\mathrm{pH}$ 8), aqueous $\mathrm{NaCl}(20 \% \mathrm{wt} /$ vol), and $0.1 \%$ sodium salt of deoxycholic acid were added to the TG and put in a boiling bath for $1 \mathrm{~min}$ at $40^{\circ} \mathrm{C}$. After the addition of $12 \mathrm{mg}$ of pancreatic swine lipase (Sigma-Aldrich, St. Louis, MO), the mixture was vigorously shaken for $1 \mathrm{~min}$ and then placed in a boiling bath and kept at $40^{\circ} \mathrm{C}$ for $15 \mathrm{~min}$. The reaction was stopped using $1 \mathrm{~mL}$ of hexane-isopropanol 3:1 ( $\mathrm{vol} /$ vol) and $1 \mathrm{~mL}$ of $4 \mathrm{M} \mathrm{HCl}$. The organic phase was separated from the aqueous phase by centrifuging the mixture at 2,500 $\times g$ for $5 \mathrm{~min}$ in a refrigerated centrifuge (Eppendorf 5804 R, Hamburg, Germany). The upper phase was withdrawn and the lipid fraction was separated by TLC as above. The spots corresponding to the phospholipids, monoglycerides, and free fatty acids were identified by comparing them with the commercial standard (Sigma-Aldrich). The monoglyceride spot was scraped with a spatula, extracted with diethyl-ether, and trans-esterified according to Christie (1982).

Methyl esters of fatty acids were separated, identified, and quantified using a GC-flame ionization detector apparatus (GC 2000 plus, Shimadzu, Columbia, MD) according to Serra et al. (2009). Conjugated linolenic acid isomers were determined by HPLC UV-diode array detector equipped with 3 assembled $\mathrm{Ag}^{2+}$ columns according to Serra et al. (2009).

\section{Fatty Acid Composition of sn-2 Position and Fatty Acid Composition of Triglycerides}

According to Pacetti et al. (2005), the fatty acid composition of monoglycerides is representative of $s n-2$ monoglycerides, considering that pancreatic lipase acts on the $s n-1$ and $s n-3$ positions of TG. Thus, the percentage of fatty acids esterified in $s n-1$ and $s n-3$ positions was obtained by applying the following formula:

$$
\begin{gathered}
\% \mathrm{FA}_{s n-1,3}(\alpha-\text { position })=\left[\left(\% \mathrm{FA}_{\mathrm{TG}} \times 3\right)\right. \\
\left.-\left(\% \mathrm{FA}_{s n-2}\right)\right] / 2
\end{gathered}
$$

where $\mathrm{FA}_{\mathrm{TG}}=$ fatty acid composition of TG, and $\mathrm{FA}_{s n-2}$ $(\beta$-position $)=$ fatty acid composition of $s n-2$.

\section{Nutritional Evaluation of sn-2 Fatty Acid Composition}

From a chemical point of view, a fatty acid can be esterified in 3 different triglyceride positions. How- ever, only 2 conditions have nutritional significance: $\alpha$ $\left(\mathrm{FA}_{s n-1,3}\right)$ and $\beta\left(\mathrm{FA}_{s n-2}\right)$ positions: $\beta$ has a preferential nutritional status compared with $\alpha$.

We postulated that an individual fatty acid is preferentially absorbed in the intestine if more than $50 \%$ of it is esterified in the $\beta$-position of triglycerides. Thus, to evaluate the putative preferential intestinal absorption index (PPIAi) of a fatty acid, we applied the following formula:

$$
\text { PPIAi }=\left\{\left[\mathrm{FA}_{s n-2} /\left(\mathrm{FA}_{s n-2}+\mathrm{FA}_{s n-1,3}\right)\right] \times 100\right\}-50 .
$$

The terms within brackets represent the percentage of single fatty acids esterified in $\beta$-position. The range of PPIAi is $0 \pm 50$; PPIAi $=0$, fatty acid with neutral nutritional condition; PPIAi $<0$, fatty acid with disadvantageous nutritional condition; and PPIAi $>0$, fatty acid with preferential nutritional condition.

\section{Statistical Analysis}

Data corresponding to the fatty acid composition of TG and PPIAi were analyzed by the following linear model:

$$
y_{j}=\mu+D_{i}+e_{i j}
$$

where $\mathrm{y}=$ dependent variable (fatty acid), $\mu=$ general mean common to all observations, $D_{i}=$ fixed effect of the ith diet with i varying from 1 to 2 (CON and LIN), and $\mathrm{e}=$ residual error.

Data corresponding to the fatty acid composition of $\mathrm{FA}_{s n-2}(\beta-)$ and $\mathrm{FA}_{s n-1,3}(\alpha-)$ were analyzed by the following mixed linear model:

$$
y_{j}=\mu+P_{j}+D_{i}+P_{j} \times D_{i}+A_{n}+e_{i j},
$$

where $\mathrm{y}=$ dependent variable (fatty acid or PPIAi), $\mu$ = general mean common to all observations, $\mathrm{P}_{\mathrm{j}}=$ fixed effect of jth lipid position with $\mathrm{j}$ varying from 1 to 2 $(s n-2$ and $s n-1,3), \mathrm{D}_{\mathrm{i}}=$ fixed effect of the ith diet with i varying from 1 to $2\left(\mathrm{CON}\right.$ and LIN), $\mathrm{A}_{\mathrm{n}}=$ random effect of nth animal, and e $=$ residual error.

Estimated means were compared by the following orthogonal contrasts: $\mathrm{CON} \beta-\mathrm{CON} \alpha=$ fatty acid composition of $s n-2(\beta-)$ versus fatty acid composition of sn-1,3 $(\alpha-)$ of the CON group; $\beta$ CON- $\beta$ LIN $=$ fatty acid composition of $s n-2(\beta-)$ of CON group versus fatty acid composition of $s n-2(\beta-)$ of the LIN group; and $\alpha \mathrm{CON}-\alpha \mathrm{LIN}=$ fatty acid composition of $s n-1,3$ $(\alpha-)$ of the CON group versus fatty acid composition of $s n-1,3(\alpha-)$ of the LIN group. 


\section{RESULTS AND DISCUSSION}

\section{Effect of Diet on Triglyceride Fatty Acid Composition}

The linseed supplementation was not a significant variation factor with respect to either the total milk lipid content (5.57 vs. $5.49 \mathrm{~g} / 100 \mathrm{~g}$ of milk in CON and LIN respectively) or the total fatty acid content, although it did affect the fatty acid composition of TG (Table 2). In fact, compared with the milk from sheep fed the CON diet, the milk from sheep fed with LIN was lower in medium-chain fatty acids (MCFA) and short-chain fatty acids (SCFA), and higher in longchain fatty acids (LCFA). In addition, as an effect of the inclusion of linseed in the diet, LIN milk provided fewer SFA $(-13 \%)$ and more PUFA $(+18 \%)$, n-3 PUFA $(+30 \%)$ and showed a lower PUFA n-6/PUFA n-3 ratio than the CON milk (Table 2).

With regard to the single fatty acid in total TG, LIN compared with CON showed a significantly higher content of both ALA (C18:3n-3) and total trans C18:1. The increase in total trans $\mathrm{C} 18: 1$ was mainly due to C18:1 trans-11 (VA). This was expected because in the rumen, ALA undergoes biohydrogenation producing, in sequence, conjugated linolenic acid (CALA; cis-9, trans11,cis-15 C18:3), trans-11, cis-15 C18:2, VA, and finally, stearic acid (C18:0). The rate-determining step in this process is the conversion from VA to stearic acid; thus, VA accumulates in the rumen (Lourenço et al., 2010). Afterward, VA is transferred to the endoplasmic reticulum of the mammary gland cells where part of it is converted into cis-9,trans-11 C18:2 (rumenic acid, RA) by stearoyl CoA-desaturase, an enzyme that inserts a cis double bond in position 9. Thus, both VA and RA were expected to be higher in LIN than in CON (Tables 2 and 3). Rumenic acid is the most bioactive CLA isomer (Kramer et al., 1998) and represented more than $70 \%$ of total CLA both in CON and LIN.

Public health authorities (e.g., the European Food Safety Authority, EFSA) have established a recommended daily allowance for different kind of fatty acids. For example, the EFSA guidelines indicate $2 \mathrm{~g} / \mathrm{d}$ for the ALA recommended daily allowance (EFSA, 2005); ALA, in fact, shows positive nutritional effects (Bhardwaj et al., 2016). Moreover, also in human tissue, VA may undergo desaturation by stearoyl CoA-desaturase and be converted to RA, thus magnifying the positive effect of RA (Jacome-Sosa et al., 2010). On the other hand, the healthy effect of CLA is well known (Rodríguez-Alcalá et al., 2017). Thus, the characteristics of LIN milk were very favorable, which means that it has a positive effect on human health. Instead, it is well known that cheesemaking does not affect the fatty acid composition and that fatty acids will be quantitatively transferred from the milk to cheese (Mele et al., 2011). In fact, a cheese made with the milk analyzed in this study reduced LDL cholesterol and some endocannabinoids such as 2-arachidonoyl glycerol and anandamide in dyslipidemic volunteers (Pintus et al., 2013).

\section{Effect of Triglyceride Positions}

The strong health effect shown by Pintus et al. (2013) may be due not only to the high milk content of VA, RA, ALA, and more generally, the n-3 fatty acids, but also to their bioavailability. Fatty acids are absorbed in the duodenum mainly as 2-monoglycerides. They are produced above all by a pancreatic lipase that hydrolyzes the fatty acids esterified in the $\alpha$-position. The fatty acids esterified in the $\beta$-position have a preferential nutritional condition and are thus preferentially and more efficiently adsorbed (Ramírez et al., 2001) than fatty acids esterified in $\alpha$-position, which can be excreted by feces.

Milk triglyceride synthesis takes place in the endoplasmic reticulum of mammary gland cells by 3 different enzymes. Glycerol-3-phosphate acyl-transferase (GPAT) catalyzes the esterification of fatty acid into $s n-1$, lyso-phosphatidic acid acyl-transferase (LPAAT) into $s n-2$, whereas diacylglycerol acyl-transferase (DGAT) "ends" triglyceride, promoting fatty acid esterification in $s n-3$. Fatty acids show a different affinity for these enzymes, resulting in a different fatty acid composition of the triglyceride positions (Yen et al., 2008).

The data in Table 4 suggest that the affinity of GPAT, LPAAT, and DGAT must be related to the acyl chain length of fatty acids, rather than the number of double bonds. In fact, if fatty acids are considered according to their acyl chain length, then SCFA and especially VFA (C4:0, C6:0, and C8:0) are preferentially esterified in $\alpha$, suggesting a higher affinity for GPAT and DGAT, whereas MCFA (mainly C12:0, C14:0) are preferentially esterified in $\beta$ (higher affinity for LPAAT). These results are in accordance with Yen et al. (2008). On the other hand, when grouping fatty acids according to their unsaturation, there were no differences in SFA and MUFA in $\alpha$ and $\beta$.

Conversely, there were differences in PUFA in the $\alpha$ and $\beta$ positions. Interestingly, we found that PUFA n- 6 and PUFA n-3 are preferentially esterified in a different position in the triglycerides (thus showing a different affinity for the aforementioned enzymes). The former (above all linoleic acid) are preferentially esterified in $\beta$, and the latter (ALA, trans-11,cis-15 C18:2 and EPA particularly) in $\alpha$ (Table 4). Another interesting result was that CLA, except for some minor isomers, did not show a preferential esterification in triglycerides (Table 
Table 2. Fatty acid composition of triglycerides as affected by treatment ( $\mathrm{g} / 100 \mathrm{~g}$ of fatty acid unless otherwise indicated $)^{1}$

\begin{tabular}{|c|c|c|c|c|}
\hline \multirow[b]{2}{*}{ Item } & \multicolumn{2}{|c|}{ Treatment } & \multirow[b]{2}{*}{$\mathrm{SE}$} & \multirow[b]{2}{*}{$P$-value } \\
\hline & $\mathrm{CON}$ & LIN & & \\
\hline Total lipids (g/100 g of milk) & 5.57 & 5.49 & 0.17 & 0.3904 \\
\hline Total fatty acids ( $\mathrm{g} / 100 \mathrm{~g}$ of total lipids) & 82.21 & 81.90 & 0.62 & 0.7356 \\
\hline C4:0 & 2.80 & 2.94 & 0.27 & 0.7272 \\
\hline C6:0 & 2.42 & 1.85 & 0.15 & 0.0139 \\
\hline $\mathrm{C} 8: 0$ & 2.11 & 1.39 & 0.16 & 0.0047 \\
\hline C10:0 & 7.10 & 4.87 & 0.71 & 0.0365 \\
\hline C10:1 cis-9 & 0.21 & 0.13 & 0.02 & 0.0152 \\
\hline $\mathrm{C} 12: 0$ & 3.55 & 2.43 & 0.21 & 0.0012 \\
\hline C13:0 & 0.06 & 0.04 & 0.01 & 0.0460 \\
\hline C13-iso & 0.06 & 0.04 & 0.01 & 0.0244 \\
\hline $\mathrm{C} 14: 0$ & 10.28 & 8.38 & 0.34 & $<0.001$ \\
\hline C14:1 cis-9 & 0.20 & 0.16 & 0.02 & 0.1178 \\
\hline C14-iso & 0.09 & 0.08 & 0.01 & 0.4333 \\
\hline C15:0 & 1.01 & 0.97 & 0.04 & 0.4526 \\
\hline C15-anteiso & 0.54 & 0.51 & 0.02 & 0.2987 \\
\hline C15-iso & 0.31 & 0.29 & 0.02 & 0.3087 \\
\hline C16:0 & 21.23 & 19.45 & 0.73 & 0.0985 \\
\hline $\mathrm{C} 16: 1$ cis-9 & 0.89 & 0.75 & 0.08 & 0.2133 \\
\hline C16:1 trans -9 & 0.25 & 0.42 & 0.07 & 0.0738 \\
\hline C16-1 cis-7 & 0.37 & 0.40 & 0.05 & 0.6761 \\
\hline C16-iso & 0.25 & 0.24 & 0.02 & 0.8249 \\
\hline $\mathrm{C} 17: 0$ & 0.60 & 0.58 & 0.02 & 0.5007 \\
\hline $\mathrm{C} 17: 1$ cis -9 & 0.17 & 0.14 & 0.02 & 0.1476 \\
\hline C17-anteiso & 0.59 & 0.50 & 0.02 & 0.0127 \\
\hline C17-iso & 0.44 & 0.43 & 0.02 & 0.7705 \\
\hline $\mathrm{C} 18: 0$ & 9.94 & 11.09 & 0.44 & 0.0817 \\
\hline C18:1 trans $-6-8$ & 0.36 & 0.56 & 0.04 & 0.0043 \\
\hline C18:1 trans -9 & 0.33 & 0.51 & 0.04 & 0.0064 \\
\hline C18:1 trans -10 & 0.52 & 0.69 & 0.05 & 0.0319 \\
\hline C18:1 trans-11 & 2.61 & 5.80 & 0.80 & 0.0101 \\
\hline $\mathrm{C} 18: 1$ trans -14 & 1.48 & 2.19 & 0.20 & 0.0207 \\
\hline C18:1 trans -15 & 0.6 & 0.74 & 0.05 & 0.0601 \\
\hline $\mathrm{C} 18: 1$ trans total & 5.93 & 10.51 & 1.04 & 0.0048 \\
\hline C18:1 cis-9 & 18.70 & 17.26 & 1.27 & 0.4301 \\
\hline C18:1 cis-11 & 0.37 & 0.42 & 0.02 & 0.0504 \\
\hline C18:1 cis-12 & 0.41 & 0.63 & 0.06 & 0.0147 \\
\hline C18:2 trans-11,cis- 15 & 1.27 & 1.78 & 0.28 & 0.2277 \\
\hline C18:2n-6 & 2.14 & 2.07 & 0.14 & 0.7208 \\
\hline C18:3n-3 & 1.89 & 2.74 & 0.16 & 0.0013 \\
\hline C18:4n-3 & 0.01 & 0.06 & 0.01 & 0.0736 \\
\hline$\alpha$-Linolenic acid & 0.02 & 0.15 & 0.04 & 0.0279 \\
\hline $\mathrm{C} 20: 0$ & 0.21 & 0.22 & 0.02 & 0.6236 \\
\hline C20:4n-6 & 0.18 & 0.11 & 0.01 & 0.0016 \\
\hline $\mathrm{C} 20: 5 n-3$ & 0.08 & 0.08 & 0.01 & 0.8745 \\
\hline $\mathrm{C} 21: 0$ & 0.06 & 0.04 & 0.01 & 0.2320 \\
\hline $\mathrm{C} 22: 0$ & 0.14 & 0.14 & 0.02 & 0.9009 \\
\hline C23:1 cis-14 & 0.05 & 0.03 & 0.01 & 0.2521 \\
\hline C24:0 & 0.04 & 0.03 & 0.01 & 0.5541 \\
\hline $\mathrm{C} 22: 5 n-3$ & 0.12 & 0.09 & 0.01 & 0.1320 \\
\hline C22:6n-3 & 0.07 & 0.04 & 0.01 & 0.0817 \\
\hline \multicolumn{5}{|l|}{ Fatty acid class } \\
\hline SFA & 63.43 & 56.12 & 1.71 & 0.0063 \\
\hline MUFA & 27.60 & 30.97 & 1.72 & 0.1790 \\
\hline PUFA & 5.81 & 7.14 & 0.38 & 0.0220 \\
\hline PUFA n-3 & 3.47 & 4.95 & 0.43 & 0.0253 \\
\hline PUFA n-6 & 2.34 & 2.20 & 0.50 & 0.4995 \\
\hline n- $6 / n-3$ & 0.79 & 0.52 & 0.09 & 0.0481 \\
\hline SCFA & 14.68 & 11.21 & 0.97 & 0.0188 \\
\hline MCFA & 39.15 & 34.18 & 1.17 & 0.0065 \\
\hline LCFA & 45.75 & 52.93 & 2.45 & 0.0408 \\
\hline
\end{tabular}

${ }^{1}$ Treatments: $\mathrm{CON}=$ control; $\mathrm{LIN}=$ linseed. $\mathrm{SCFA}=$ short-chain fatty acids (acyl chain less than 11 carbon atoms); $\mathrm{MCFA}=$ medium-chain fatty acids (acyl chain more than 10 carbon atoms and less than 17 carbon atoms); LCFA $=$ long-chain fatty acids (acyl chain more than 16 carbon atoms). 
Table 3. Conjugated linoleic acid triglyceride isomers as affected by treatment $(\mathrm{mg} / 100 \mathrm{~g} \text { of fatty acid })^{1}$

\begin{tabular}{lrrrr}
\hline & \multicolumn{2}{c}{ Treatment } & & \\
\cline { 2 - 3 } Item & CON & LIN & SE & P-value \\
\hline $11 / 13$ cis/trans & 1.87 & 0.89 & 3.14 & 0.8418 \\
$11 / 13$ trans/cis & 51.95 & 218.96 & 31.94 & 0.0012 \\
$11 / 13$ trans/trans & 72.59 & 106.78 & 13.58 & 0.0801 \\
$12 / 14$ cis/trans & 35.52 & 54.51 & 11.77 & 0.1914 \\
$12 / 14$ trans/trans & 50.45 & 68.09 & 10.27 & 0.1892 \\
$7 / 9$ trans/cis & 37.67 & 62.25 & 10.54 & 0.2630 \\
$7 / 9$ trans/trans & 8.12 & 10.32 & 3.82 & 0.3892 \\
$8 / 10$ cis/trans & 69.52 & 88.88 & 17.63 & 0.1564 \\
$8 / 10$ trans/trans & 11.27 & 8.38 & 4.44 & 0.9643 \\
$9 / 11$ cis/trans $(\mathrm{RA})$ & $1,549.38$ & $2,711.27$ & 266.06 & $<0.001$ \\
$9 / 11$ trans/trans & 46.48 & 54.31 & 7.43 & 0.1155 \\
Total cis/trans or trans/cis & $1,745.91$ & $3,136.76$ & 304.97 & $<0.001$ \\
Total CLA & $1,956.44$ & $3,394.94$ & 316.60 & $<0.001$ \\
Total trans/trans & 210.54 & 258.18 & 26.32 & 0.0756 \\
\hline
\end{tabular}

${ }^{1}$ Treatments: $\mathrm{CON}=$ control; $\mathrm{LIN}=$ linseed. $\mathrm{RA}=$ rumenic acid

$5)$; in particular, RA showed a very similar distribution between $\alpha$ and $\beta$ positions. Thus, the CON milk showed a fatty acid composition of triglycerides that does not facilitate the absorption of the most healthy fatty acids. In fact, as RA, VA did not show a preferential triglyceride position, and PUFA n-3 was preferentially esterified in $s n-1,3$. Finally, myristic acid (C14:0), the unhealthiest fatty acid (Zock et al., 2016), was preferentially esterified in $s n-2$, whereas palmitic acid, another unhealthy fatty acid, showed no preferential esterification (Table 4).

\section{Effect of the Diet on the Fatty Acid Composition of Triglyceride Positions}

Table 4 also shows the effects of linseed supplementation on the fatty acid composition of the $\alpha$ - and $\beta$-triglyceride positions. Interestingly, the diet was a significant variation factor above all in terms of the fatty acid composition of the $\beta$-position. As a consequence of the linseed supplementation, sn-2 was lower in SFA $(-19 \%)$ and higher in MUFA $(+27 \%)$, PUFA $(+48 \%)$, and PUFA n-3 $(+126 \%)$, whereas the diet did not affect the fatty acid classes of $\alpha$-position.

If we consider single fatty acids, with linseed supplementation, ALA increased both in $\beta$ - and $\alpha$-positions, but above all, in $\beta$-position (more than 93\%, whereas in $\alpha$-position it only increased by $33 \%$ ), VA also increased in $s n-2$ by more than $280 \%, \mathrm{C} 18: 2$ trans- 11 , cis- 15 by more than $283 \%$, and CALA by more than $1,700 \%$. Conversely, we observed a significant decrease in lauric acid (C12:0; $-32 \%)$, myristic acid (C14:0; $-18 \%)$, and palmitic acid $(\mathrm{C} 16: 0 ;-29 \%)$ for the same triglyceride position. As a consequence of the increase in n-3 PUFA, the n- $6 / \mathrm{n}-3$ ratio of $s n-2$ position was significantly lower in LIN than in $s n-1,3$.
The effect of linseed supplementation on CLA isomers was mostly for the $s n-2$ position. In fact, total CLA increased by more than 2.4 times in $s n-2$ and only 1.5 times in $s n-1,3$. This effect is mainly due to RA (which was over $75 \%$ of total CLA); RA was more 2.6 times in $s n-2$ triglycerides from milk from animals fed LIN with respect to the CON, whereas its content in $s n-1,3$ was not affected by the diet (Table 5 ).

These results led us to hypothesize that the affinity for LPAAT of ALA, CALA, trans-11,cis-15 C18:2, VA, and RA dramatically increases as they become more available in tissues.

\section{Effect of the Diet on PPIAi of Fatty Acids}

The fatty acid position in triglycerides may affect their bioavailability, however, in contrast to the chemical perspective [i.e., a fatty acid can be esterified in 3 different positions ( $s n-1, s n-2$, and $s n-3)]$, the nutritional condition of a fatty acid is determined only by being esterified in $\alpha\left(\mathrm{FA}_{s n-1,3}\right)$ or in $\beta\left(\mathrm{FA}_{s n-2}\right)$. The $\beta$-position represents a preferential nutritional condition with respect to the $\alpha$. We assumed that a fatty acid will have a preferential intestinal absorption if more than 50\% of it is esterified in $\beta$-position. We estimated this by a PPIAi (Figures 1 and 2). The PPIAi variation field is $0 \pm 50$; fatty acids with a PPIAi higher than 0 will be putatively preferentially absorbed, whereas fatty acids with a PPIAi value less than 0 show a disadvantageous absorption condition. Thus, the PPIAi of a fatty acid can be considered as a proxy of its bioavailability.

Linseed supplementation dramatically affected PPIAi and, as a result, the fatty acid bioavailability. Figure 1 shows the PPIAi of fatty acid classes, Figure 2 includes the PPIAi of selected fatty acids, and PPIAi of the other fatty acids are shown in Supplemental Figures 
Table 4. Fatty acid composition of $s n-2$ and $s n-1,3$ monoglycerides as affected by treatment $(\mathrm{g} / 100 \mathrm{~g} \text { of fatty acid unless otherwise indicated })^{1}$

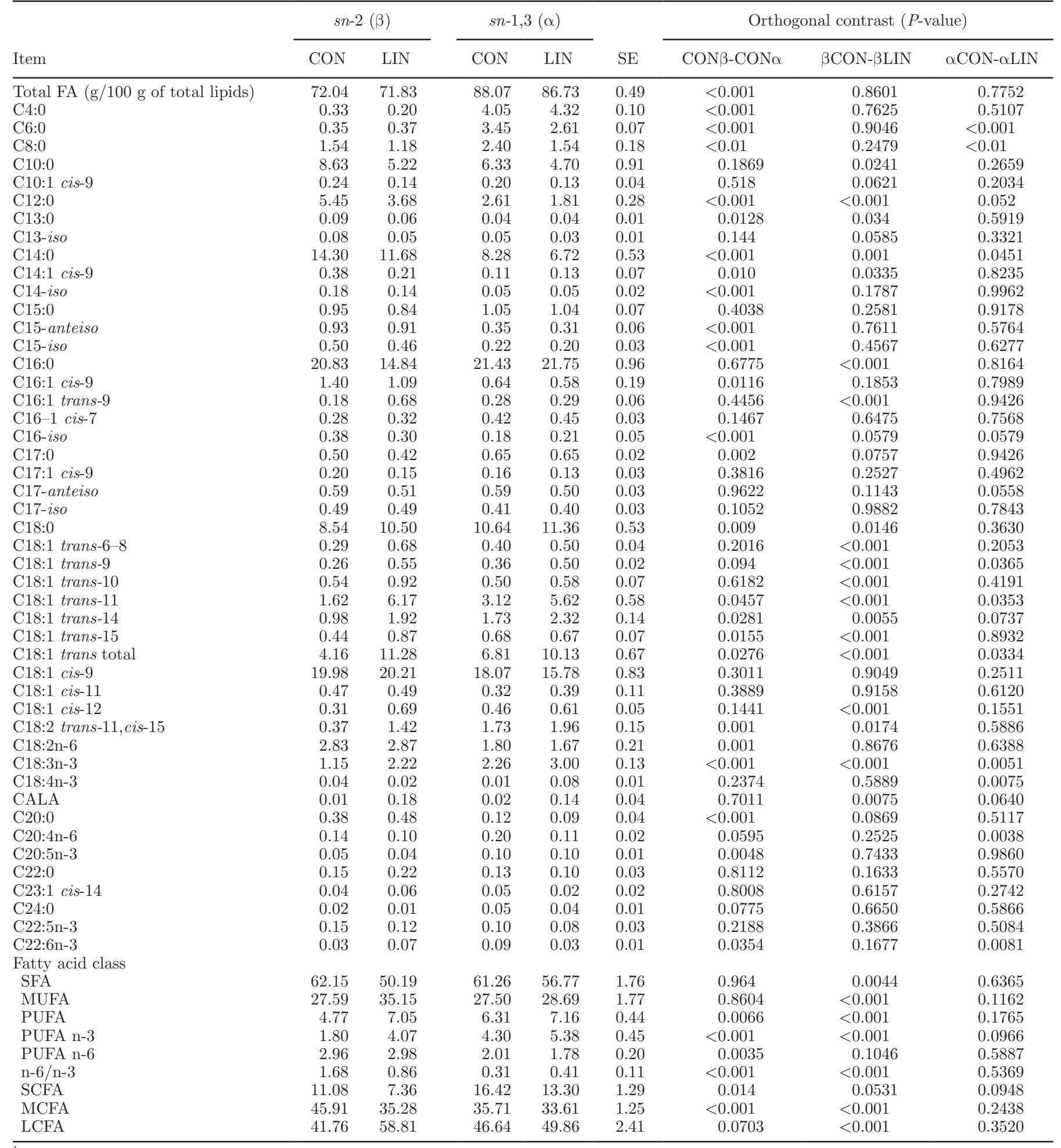

${ }^{1}$ Treatments: $\mathrm{CON}=$ control; LIN $=$ linseed. CALA = conjugated linolenic acid; SCFA = short-chain fatty acids (acyl chain less than 11 carbon atoms); $\mathrm{MCFA}=$ medium-chain fatty acids (acyl chain more than 10 carbon atoms and less than 17 carbon atoms); LCFA = long-chain fatty acids (acyl chain more than 16 carbon atoms). $s n-2=2$-monoglyceride; $s n-1,3=1$-monoglyceride +3 -monoglyceride. 


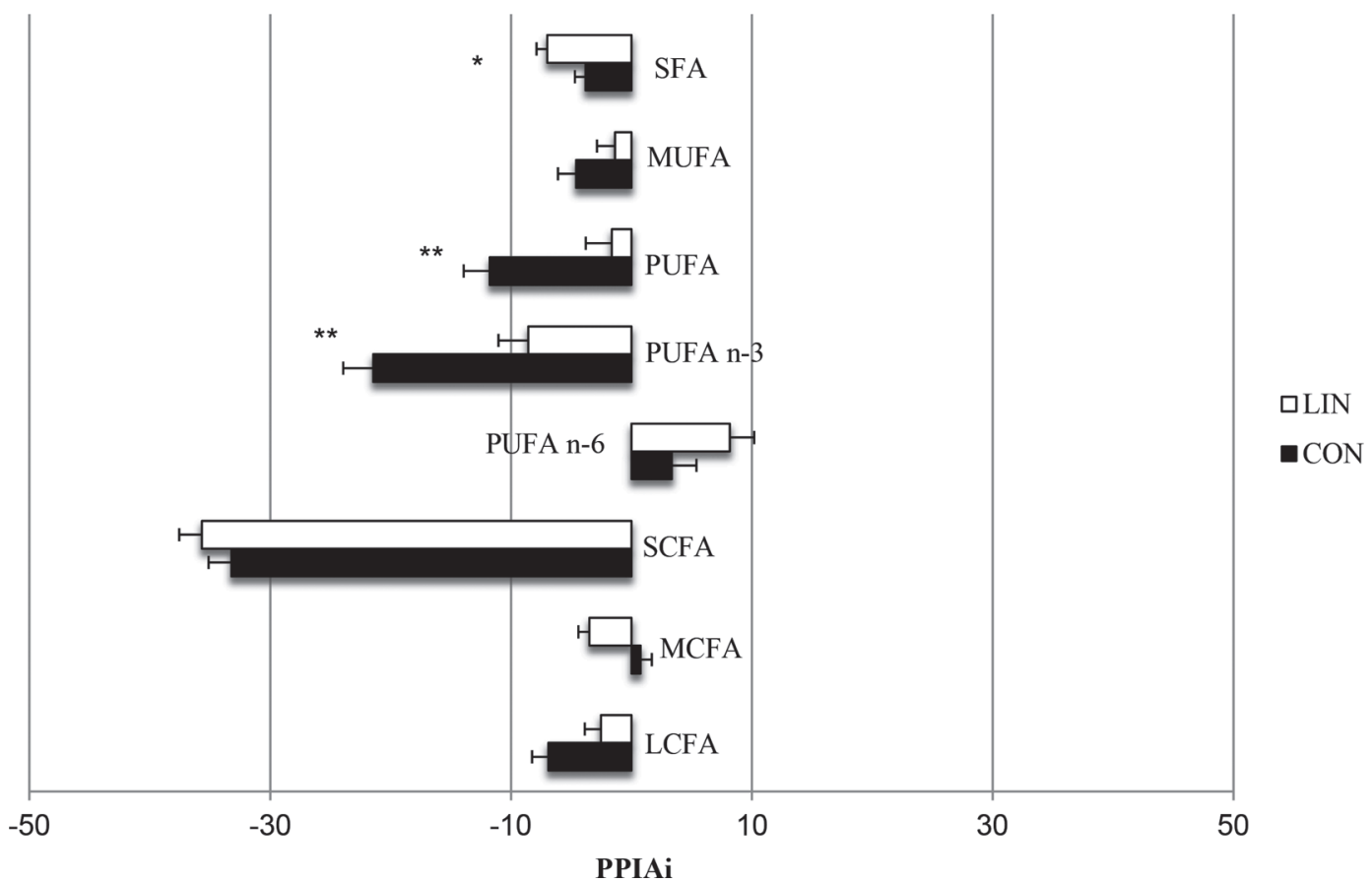

Figure 1. Putative preferential intestinal absorption index (PPIAi) of fatty acid classes. CON $=$ control; LIN $=$ linseed. PPIAi $=\left\{\left[\mathrm{FA}_{s n-2} /\right.\right.$ $\left.\left.\left(\mathrm{FA}_{s n-2}+\mathrm{FA}_{s n-1,3}\right)\right] \times 100\right\}-50 ; \mathrm{FA}_{s n-2}=s n-2$ fatty acid content; $\mathrm{FA}_{s n-1,3}=s n-1,3$ fatty acid content. PPIAi $=0$, fatty acid with neutral nutritional condition: PPIAi $<0$, fatty acid with a disadvantageous nutritional status; PPIAi $>0$, fatty acid with a preferential nutritional status $\mathrm{SCFA}=$ short-chain fatty acids (acyl chain less than 11 carbon atoms); MCFA = medium-chain fatty acids (acyl chain more than 10 carbon atoms and less than 17 carbon atoms); LCFA = long-chain fatty acids (acyl chain more than 16 carbon atoms). ${ }^{*} 0.01 \leq P<0.05 ; * * 0.001 \leq$ $P<0.01$. Error bars indicate SE.

S1-S4 (https://doi.org/10.3168/jds.2017-14188). With respect to fatty acid classes, diet affected the PPIAi of MCFA and LCFA. In particular, in milk from sheep fed LIN, the $\beta$-position of triglycerides was higher in LCFA (PPIAi less negative than CON), and lower in MCFA
(PPIAi more negative than CON); the PPIAi of SCFA was not affected by treatment (Figure 1).

The effect on LCFA is mainly due to PUFA and particularly to PUFA n-3 (Figure 1). With respect to the fatty acids belonging to the $n-3$ series, the most af-

Table 5. Conjugated linoleic acid isomers of $s n-2$ and $s n-1,3$ monoglycerides as affected by treatment $(\mathrm{mg} / 100 \mathrm{~g} \text { of fatty acid })^{1}$

\begin{tabular}{|c|c|c|c|c|c|c|c|c|}
\hline \multirow[b]{2}{*}{ Item } & \multicolumn{2}{|c|}{$s n-2(\beta)$} & \multicolumn{2}{|c|}{$s n-1,3(\alpha)$} & \multirow[b]{2}{*}{$\mathrm{SE}$} & \multicolumn{3}{|c|}{ Orthogonal contrast $P$-value } \\
\hline & $\mathrm{CON}$ & LIN & $\mathrm{CON}$ & LIN & & $\mathrm{CON} \beta-\mathrm{CON} \alpha$ & $\beta \mathrm{CON}-\beta \mathrm{LIN}$ & $\alpha \mathrm{CON}-\alpha \mathrm{LIN}$ \\
\hline $\begin{array}{l}\text { Total FA } \\
\text { (g/100 g of fraction) }\end{array}$ & 72.04 & 71.83 & 88.07 & 86.73 & 0.49 & $<0.001$ & 0.8601 & 0.7752 \\
\hline $10 / 12$ trans $/$ trans & 5.44 & 7.35 & 26.58 & 7.38 & 2.24 & 0.0857 & 0.8106 & 0.1000 \\
\hline $11 / 13$ trans $/$ cis & 18.83 & 133.67 & 68.51 & 261.61 & 19.25 & 0.4628 & 0.0737 & 0.0022 \\
\hline $11 / 13$ trans $/$ trans & 100.16 & 112.87 & 58.80 & 103.74 & 17.25 & 0.1682 & 0.5766 & 0.0545 \\
\hline $12 / 14$ cis/trans & 16.71 & 37.17 & 44.92 & 63.19 & 9.22 & 0.3822 & 0.3920 & 0.3006 \\
\hline $12 / 14$ trans $/$ trans & 65.97 & 73.76 & 42.69 & 65.25 & 14.28 & 0.2430 & 0.6666 & 0.1653 \\
\hline $7 / 9$ trans $/$ cis & 11.54 & 9.55 & 50.73 & 88.59 & 2.42 & 0.0349 & 0.8950 & 0.0298 \\
\hline $7 / 9$ trans $/$ trans & 17.25 & 25.79 & 3.56 & 2.59 & 5.53 & 0.1429 & 0.1528 & 0.9119 \\
\hline $8 / 10$ cis/trans & 69.54 & 134.98 & 69.50 & 65.83 & 15.44 & 0.5779 & 0.0866 & 0.7588 \\
\hline $8 / 10$ trans/trans & 16.66 & 21.32 & 8.58 & 1.91 & 6.95 & 0.0996 & 0.5607 & 0.5541 \\
\hline $9 / 11$ cis/trans $(\mathrm{RA})$ & $1,149.30$ & $3,011.04$ & $1,749.42$ & $2,561.38$ & 383.68 & 0.4902 & 0.0018 & 0.0588 \\
\hline $9 / 11$ trans/trans & 30.63 & 60.51 & 54.41 & 51.21 & 10.62 & 0.3917 & 0.0474 & 0.8108 \\
\hline Total cis/trans or trans/cis & $1,270.45$ & $3,337.04$ & $1,983.64$ & $3,036.62$ & 413.25 & 0.4582 & 0.0026 & 0.0396 \\
\hline Total trans/trans & 243.22 & 304.51 & 194.19 & 235.01 & 34.08 & 0.2720 & 0.2059 & 0.2101 \\
\hline Total CLA & $1,513.67$ & $3,641.55$ & $2,177.83$ & $3,271.64$ & 422.50 & 0.5564 & 0.0027 & 0.0375 \\
\hline
\end{tabular}

${ }^{1}$ sn-2 = 2-monoglyceride; $s n-1,3=1$-monoglyceride +3 -monoglyceride; RA = rumenic acid. Treatments: CON = control; LIN = linseed. 
fected PPIAi was ALA and, in a broad sense, the fatty acids from its rumen biohydrogenation, such as CALA (cis-9,trans-11,cis-15 C18:3), trans-11,cis-15 C18:2, trans-VA (trans-11 C18:1), and obviously, RA (Figure 2 ). Linseed supplementation led to an increase in the PPIAi of ALA, CALA, and trans-11,cis-15 C18:2, which was more evident for CALA (the PPIAi of CON was negative, whereas the PPIAi of LIN was positive). The effect of diet was striking for VA and RA; in milk from animals fed the CON diet, the PPIAi was less than 0 , thus showing a disadvantageous absorption condition. On the other hand, as a consequence of linseed supplementation, VA and RA PPIAi was $>0$, thus in a putative preferential intestinal absorption condition.

The PPIAi of SFA was also affected by lipid supplementation (Figure 1); however, as opposed to the aforementioned fatty acids, the PPIAi of the SFA of animals fed linseed was significantly lower than animals fed the CON diet, indicating a decrease in intestinal putative absorption as a consequence of linseed supplementation. This evidence is attributable to palmitic acid, which was the only SFA showing a PPIAi significantly affected by diet (Figure 2). This is an interesting result, given that in milk, the C16:0 was not affected by diet, whereas the C10:0, lauric, and myristic acids were af- fected (Table 2). Therefore, with respect to SFA, unlike PUFA, the affinity for LPAAT may perhaps not be related to their tissue availability.

This is a favorable scenario, as the linseed supplementation increased the bioavailability of the healthiest fatty acids, decreased the bioavailability of the unhealthiest fatty acids, and generally improved health properties of milk. These findings may provide a convincing explanation for the strong positive effect of this kind of milk on human health (Pintus et al., 2013).

\section{CONCLUSIONS}

The results of this study indicate that linseed supplementation was effective in improving the nutritional properties of milk lipids, both via a modification of the fatty acid composition and their bioavailability. In fact, milk triglycerides from animals fed linseed, with respect to the CON diet, was higher in the healthiest fatty acid (ALA, VA, and RA), and conversely, lower in the unhealthiest ones (myristic acid). The results of this study also demonstrate that the aforementioned modification was mainly related to the $s n-2$ triglyceride position. It is also interesting that, probably due to changes in their acyl-transferase enzyme (LPAAT) selectivity, the

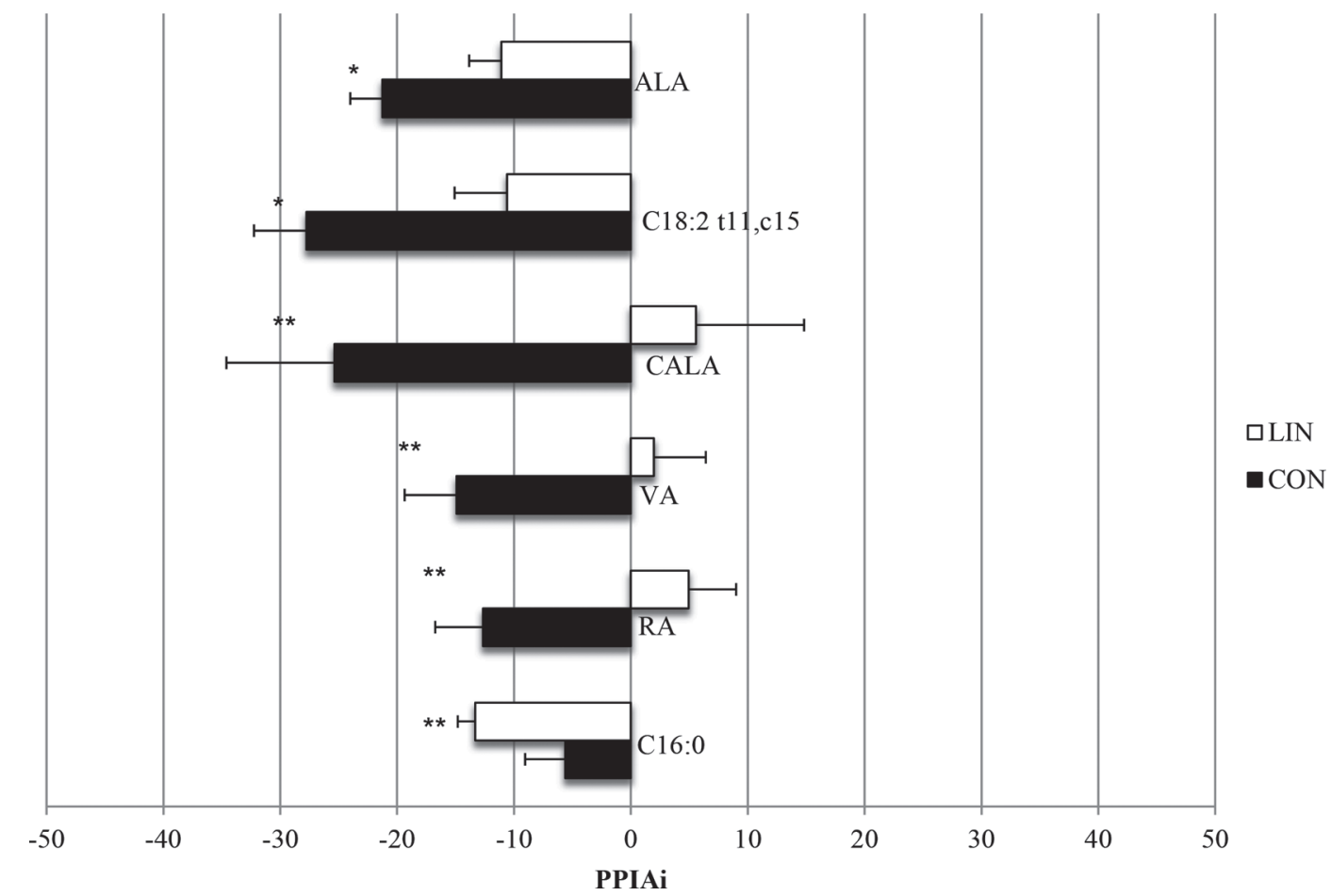

Figure 2. Putative preferential intestinal absorption index (PPIAi) of selected fatty acids. CON $=$ control; $\mathrm{LIN}=$ linseed. PPIAi $=\left\{\left[\mathrm{FA} \mathrm{s}_{n-2} /\right.\right.$ $\left.\left.\left(\mathrm{FA}_{s n-2}+\mathrm{FA}_{s n-1,3}\right)\right] \times 100\right\}-50 ; \mathrm{FA}_{s n-2}=s n-2$ fatty acid content; $\mathrm{FA}_{s n-1,3}=s n-1,3$ fatty acid content. PPIAi $=0$, fatty acid with neutral nutritional condition; PPIAi $<0$, fatty acid with a disadvantageous nutritional status; PPIAi $>0$, fatty acid with a preferential nutritional status. $\mathrm{ALA}=\alpha$-linolenic acid; $\mathrm{t}=$ trans; $\mathrm{c}=$ cis $; \mathrm{CALA}=$ conjugated linolenic acid; $\mathrm{VA}=$ vaccenic acid; $\mathrm{RA}=$ rumenic acid. ${ }^{*} 0.01 \leq P<0.05 ;$ $* * 0.001 \leq P<0.01$. Error bars indicate SE. 
linseed supplementation affected the PPIAi of the fatty acids, and thus their bioavailability. This thus confirms that linseed supplementation is an effective and economically sustainable approach to improving the health properties of milk.

\section{ACKNOWLEDGMENTS}

This research was supported by Personal University Fund (University of Pisa, Pisa, Italy).

\section{REFERENCES}

Bhardwaj, K., V. Narsingh, R. K. Trivedi, S. Bhardwaj, and N. Shukla. 2016. Significance of ratio of omega-3 and omega- 6 in human health with special reference to flaxseed oil. Int. J. Biol. Chem. $10: 1-6$.

Blondeau, N. 2016. The nutraceutical potential of omega-3 alphalinolenic acid in reducing the consequences of stroke. Biochimie 120:49-55.

Chen, L., G. E. Remondetto, and M. Subirate. 2006. Food proteinbased materials as nutraceutical delivery system. Trends Food Sci. Technol. 17:272-283.

Christie, W. W. 1982. A simple procedure of rapid transmethylation of glycerolipids and cholesteryl esters. J. Lipid Res. 23:1072-1075.

Dawczynski, C., L. Martin, A. Wagner, and G. Jahreis. 2010. N-3 LC-PUFA-enriched dairy products are able to reduce cardiovascular risk factors: A double-blind, cross-over study. Clin. Nutr. 29:592-599.

Dawczynski, C., K. A. Massey, C. Ness, M. Kiehntopf, S. Stepanow, M. Platzer, M. Grün, A. Nicolaou, and G. Jahreis. 2013. Randomized placebo-controlled intervention with n-3 LC-PUFA- supplemented yoghurt: Effects on circulating eicosanoids and cardiovascular risk factors. Clin. Nutr. 32:686-696.

EFSA. 2005. Opinion of the Scientific Panel on Dietetic Products, Nutrition and Allergies on a request from the Commission related to nutrition claims concerning omega-3 fatty acids, monounsaturated fat, polyunsaturated fat and unsaturated fat. EFSA J. 253:1-29.

Ghazal, S., V. Berthelot, N. C. Friggens, and P. Schmidely. 2014. Effect of conjugated linoleic acid supplementation and feeding level on dairy performance, milk fatty acid composition, and body fat changes in mid-lactation goats. J. Dairy Sci. 97:7162-7174.

Jacome-Sosa, M. M., J. Lu, Y. Wang, M. R. Ruth, D. C. Wright, M. J. Reaney, J. Shen, C. J. Field, D. F. Vine, and S. D. Proctor. 2010. Increased hypolipidemic benefits of cis-9, trans-11 conjugated linoleic acid in combination with trans-11 vaccenic acid in a rodent model of the metabolic syndrome, the JCR:LA-cp rat. Nutr. Metab. (Lond.) 7:60.
Kramer, J. K. G., P. W. Parodi, R. G. Jensen, M. M. Mossoba, M. P. Yurawecz, and R. O. Adlof. 1998. Rumenic acid: A proposed common name for the major conjugated linoleic acid isomer found in natural products. Lipids 33:835.

Lourenço, M., E. Ramos-Morales, and R. J. Wallace. 2010. The role of microbes in rumen lipolysis and biohydrogenation and their manipulation. Animal 4:1008-1023.

Mele, M., G. Contarini, L. Cercaci, A. Serra, A. Buccioni, M. Povolo, G. Conte, A. Funaro, S. Banni, G. Lercker, and P. Secchiari. 2011. Enrichment of Pecorino cheese with conjugated linoleic acid by feeding dairy ewes with extruded linseed: Effect on fatty acid and triglycerides composition and on oxidative stability. Int. Dairy J. $21: 365-372$.

Mu, H., and C. E. Høy. 2004. The digestion of dietary triacylglycerols. Prog. Lipid Res. 43:105-133.

Pacetti, D., H. W. Hulan, M. Schreiner, E. Boselli, and N. G. Frega. 2005. Positional analysis of egg triacylglycerols and phospholipids from hens fed diets enriched with refined seal blubber oil. J. Sci. Food Agric. 85:1703-1714.

Pintus, S., E. Murru, G. Carta, L. Cordeddu, B. Batetta, S. Accossu, D. Pistis, S. Uda, M. E. Ghiani, M. Mele, P. Secchiari, G. Almerighi, P. Pintus, and S. Banni. 2013. Sheep cheese naturally enriched in $\alpha$-linolenic, conjugated linoleic and vaccenic acids improves the lipid profile and reduces anandamide in the plasma of hypercholesterolaemic subjects. Br. J. Nutr. 109:1453-1462.

Ramírez, M., L. Amate, and A. Gil. 2001. Absorption and distribution of dietary fatty acids from different sources. Early Hum. Dev. 65:S95-S101.

Rodríguez-Alcalá, L. M., M. P. Castro-Gómez, L. L. Pimentel, and J. Fontecha. 2017. Milk fat components with potential anticancer activity-A review. Biosci. Rep. 37:BSR20170705.

Santini, A., G. C. Tenore, and E. Novellino. 2017. Nutraceuticals: A paradigm of proactive medicine. Eur. J. Pharm. Sci. 96:53-61.

Serra, A., M. Mele, F. La Comba, G. Conte, A. Buccioni, and P. Secchiari. 2009. Conjugated linoleic acid (CLA) content of meat from three muscles of Massese suckling lambs slaughtered at different weights. Meat Sci. 81:396-404.

Sofi, F., A. Buccioni, F. Cesari, A. M. Gori, S. Minieri, L. Mannini, A. Casini, G. F. Gensini, R. Abbate, and M. Antongiovanni. 2010. Effects of a dairy product (pecorino cheese) naturally rich in cis9, trans-11 conjugated linoleic acid on lipid, inflammatory and haemorheological variables: A dietary intervention study. Nutr. Metab. Cardiovasc. Dis. 20:117-124.

Yen, C. L. E., S. J. Stone, S. Koliwad, C. Harris, and R. V. Farese. 2008. DGAT enzymes and triacylglycerol biosynthesis. J. Lipid Res. 49:2283-2301.

Zock, P. L., W. A. M. Blom, J. A. Nettleton, and G. Hornstra. 2016. Progressing insights into the role of dietary fats in the prevention of cardiovascular disease. Curr. Cardiol. Rep. 18:111. 